Check for updates

Cite this: RSC Adv., 2019, 9, 2885

Received 13th November 2018

Accepted 7th January 2019

DOI: $10.1039 / c 8 r a 09351 b$

rsc.li/rsc-advances

\section{Production of rhamnolipids with different proportions of mono-rhamnolipids using crude glycerol and a comparison of their application potential for oil recovery from oily sludge}

\author{
Feng Zhao, (D) *abc Hao Jiang, ${ }^{\text {bc }}$ Huichun Sun, ${ }^{a}$ Chang Liu, ${ }^{d}$ Siqin Han ${ }^{a}$ \\ and Ying Zhang*a
}

\begin{abstract}
The use of efficient green cleaning agents, such as biosurfactants, is important in oil sludge treatment. Enhanced oil recovery from oily sludge by different rhamnolipids was comparatively evaluated. Using crude glycerol, the wild-type strain Pseudomonas aeruginosa SG and the recombinant strains $P$. aeruginosa PrhlAB and $P$. stutzeri Rhl produced $1.98 \mathrm{~g} \mathrm{~L}^{-1}, 2.87 \mathrm{~g} \mathrm{~L}^{-1}$ and $0.87 \mathrm{~g} \mathrm{~L}^{-1}$ of rhamnolipids, respectively. The three bacterial strains produced different rhamnolipid mixtures under the same conditions. The proportions of mono-rhamnolipids in the three rhamnolipid products were $55.92 \%$, $94.92 \%$ and $100 \%$, respectively. These rhamnolipid products also possessed different bioactivities. Emulsifying activity became higher as the proportion of mono-rhamnolipids increased. The three rhamnolipid products were stable at temperatures lower than $121{ }^{\circ} \mathrm{C}, \mathrm{pH}$ values from $5-11$ and $\mathrm{NaCl}$ concentrations from $0-15 \%$. All three rhamnolipid products could recover oil from oily sludge, but oil recovery efficiency was positively related to the proportion of mono-rhamnolipids. Mono-rhamnolipids produced by the recombinant strain Rhl exhibited the best oil recovery efficiency $(53.81 \%)$. The results reveal that mono-rhamnolipids are the most promising for oil recovery from oily sludge.
\end{abstract}

\section{Introduction}

A large amount of oily sludge (solid waste containing oil) is produced in the process of oil production, transportation, refining and treatment of oily sewage. ${ }^{1,2}$ Oily sludge has become a major source of soil oil pollution. ${ }^{1}$ Oily sludge has been included in the national hazardous waste list in China. Oily sludge is the primary pollutant from the oil industry. ${ }^{3}$ Although oily sludge is a solid waste product, it is also a high oil content resource. ${ }^{4,5}$ How to recycle crude oil from sludge in an environmentally friendly and economically efficient way is significant to the treatment of oily sludge.

Surfactant-enhanced sludge cleaning is the focus of sludge treatment technology. 6 , This mainly refers to the use of surfactant for desorption and emulsification of crude oil in oily sludge. ${ }^{6}$ Then, separation of oil and sludge is completed under

${ }^{a}$ CAS Key Laboratory of Pollution Ecology and Environmental Engineering, Institute of Applied Ecology, Chinese Academy of Sciences (CAS), Shenyang, Liaoning Province, 110016, China. E-mail: zhao2008569@126.com; yzhang@iae.ac.cn; Tel: +86-24-83970370

${ }^{b}$ Heilongjiang Weikaier Biotechnology Company Limited, Harbin, Heilongjiang Province, 150025, China

${ }^{c}$ Harbin Hongda Construction Development Group, Harbin, Heilongjiang Province, 150000, China

${ }^{d}$ School of Environmental Science, Liaoning University, Shenyang 110036, China the action of an external force, such as centrifugal force. Surfactants are widely used and the consumption of surfactants is increasing. However, the residual chemical surfactants are not easily biodegraded..$^{89}$ So they may cause secondary pollution. ${ }^{9}$ The consequent damage to the ecosystem has been paid more and more attention.

Compared with chemical surfactants, biosurfactants have good environmental compatibility, high surface activity and easy biodegradability. ${ }^{10,11}$ The emulsifying activity of biosurfactant and the formation of micelles can disperse and solubilize petroleum hydrocarbons. ${ }^{\mathbf{1 2}}$ In oily sludge washing treatment, biosurfactants with high emulsification activity play a stronger role in solubilization of crude oil. The chemical structure of biosurfactants is diverse, which makes it possible to obtain biosurfactants with different activities. ${ }^{13-15}$ Rhamnolipids are one of the most popular biosurfactants. Rhamnolipids are a series of homologues composed of rhamnoses and fatty acids. ${ }^{16,17}$ Different rhamnolipid-producing bacterial strains afford rhamnolipid products with different structures. ${ }^{18}$ The different structures of rhamnolipids may give quite different physico-chemical properties, such as emulsifying activity. ${ }^{\mathbf{1 4 , 1 9}}$

In this study, three different rhamnolipid producers (Pseudomonas aeruginosa SG, P. aeruginosa PrhlAB, P. stutzeri Rhl) were used for rhamnolipid production. Crude glycerol was used as the low-cost substrate. Three different rhamnolipid products 
were extracted. The structural compositions and surface-active properties of the three rhamnolipids products were characterized. The rhamnolipids' stability against environmental stresses was evaluated at different temperatures, $\mathrm{pH}$ values and salinities. Further, the application potential of the three kinds of rhamnolipid products for oily sludge washing was also comparatively investigated.

\section{Materials and methods}

\section{Strains}

In this study, three different rhamnolipid producers (Pseudomonas aeruginosa SG, $P$. aeruginosa PrhlAB, $P$. stutzeri $\mathrm{Rhl}$ ) were used for rhamnolipid production. Strain SG is the wild-type strain, which was isolated from production water of Xinjiang oil reservoir, China. ${ }^{20}$ Strain PrhlAB is a recombinant strain derived from strain SG by increasing the copy numbers of the rhlAB genes. ${ }^{21}$ Strain $\mathrm{Rhl}$ is also a recombinant strain, constructed by heterologous expression of rhlABRI genes in strain $P$. stutzeri DQ1. ${ }^{22}$

\section{Medium and culture conditions}

Luria-Bertani (LB) medium was used to prepare seed culture. The three strains were cultured at $37^{\circ} \mathrm{C}$ and $200 \mathrm{rpm}$ for $16 \mathrm{~h}$. The medium used for rhamnolipid production contained $60 \mathrm{~g}$ $\mathrm{L}^{-1}$ crude glycerol, $3.4 \mathrm{~g} \mathrm{~L}^{-1} \mathrm{KH}_{2} \mathrm{PO}_{4}, 4.0 \mathrm{~g} \mathrm{~L}^{-1} \mathrm{~K}_{2} \mathrm{HPO}_{4} \cdot 3 \mathrm{H}_{2} \mathrm{O}$, $0.80 \mathrm{~g} \mathrm{~L}^{-1} \mathrm{MgSO}_{4} \cdot 7 \mathrm{H}_{2} \mathrm{O}, 3.5 \mathrm{~g} \mathrm{~L}^{-1} \mathrm{NaNO}_{3}, 0.50 \mathrm{~g} \mathrm{~L}^{-1} \mathrm{KCl}, 0.05 \mathrm{~g}$ $\mathrm{L}^{-1} \mathrm{CaCl}_{2}, 0.50 \mathrm{~g} \mathrm{~L}^{-1} \mathrm{NaCl}$. The $\mathrm{pH}$ of the medium was adjusted to 6.8 using $1 \mathrm{M} \mathrm{NaOH}$. Crude glycerol was purchased from an agency company in Jinan, China. This crude glycerol contained 90\% glycerol, some water, methanol, and esters. The other chemicals were analytical grade, and were purchased from Sinopharm Chemical Reagent Co., Ltd, China. The culture conditions for rhamnolipid production were $37{ }^{\circ} \mathrm{C}, 200 \mathrm{rpm}$ for 5 days. The inoculum amount of seed culture was $3 \%(\mathrm{v} / \mathrm{v})$. In cultivation experiments, $250 \mathrm{~mL}$ Erlenmeyer flasks containing $120 \mathrm{~mL}$ medium were used.

\section{Rhamnolipid extraction}

Cultures of the three strains were centrifuged at $10000 \mathrm{rpm}$ for $10 \mathrm{~min}$. Cell-free culture was collected and heated at $80^{\circ} \mathrm{C}$ for $30 \mathrm{~min}$. The soluble protein in cell-free culture degenerated and precipitated. Treated cell-free culture was centrifuged at $10000 \mathrm{rpm}$ for $10 \mathrm{~min}$ again. The supernatant was collected. The $\mathrm{pH}$ of the supernatant was adjusted to 1.5 using $6 \mathrm{M} \mathrm{HCl}$. The supernatant was placed at $4{ }^{\circ} \mathrm{C}$ for $16 \mathrm{~h}$. Then the sample was centrifuged at $10000 \mathrm{rpm}$ for $10 \mathrm{~min}$. The precipitate was collected and then dissolved in $0.1 \mathrm{M} \mathrm{NaHCO}_{3}$. The rhamnolipid product in $\mathrm{NaHCO}_{3}$ solution was extracted using chloroform/methanol (v/v, $2: 1)$. The extraction solution was dried by vacuum rotary evaporation $\left(65^{\circ} \mathrm{C}, 50 \mathrm{rpm}\right)$. The obtained yellow solid substance was the rhamnolipid product.

\section{Quantitative analysis by oil-spreading method}

Rhamnolipid concentrations in the three bacterial cultures were quantified by the oil-spreading method. ${ }^{23}$ The three rhamnolipid products were respectively dissolved in distilled water. Rhamnolipids-water solutions were prepared with different concentrations $(100,200,300,400,500,600,700$ and $800 \mathrm{mg} \mathrm{L}^{-1}$ ). The oil-spreading circle diameters of the rhamnolipid solutions were measured as previously described. ${ }^{23}$ Standard curves of oil-spreading circle diameters and rhamnolipid concentrations were prepared. Linear correlations were established between the oil-spreading circle diameters and rhamnolipid concentrations. The oil-spreading circle diameters of the bacterial cultures were measured. The rhamnolipid concentrations in the three bacteria cultures were calculated using the related standard curves.

\section{Qualitative analysis by TLC and FTIR}

Thin-layer chromatography (TLC) analysis was performed according to previous studies. ${ }^{\mathbf{2 0} 22}$ The three rhamnolipid products were respectively dissolved in methanol to a concentration of $200 \mathrm{mg} \mathrm{L}^{-1}$. Then, $10 \mu \mathrm{L}$ of sample was spotted on silica gel G plates (Qingdao Marine Chemical Factory, Qingdao, China). Chloroform/methanol/distilled water (90:25:2, v/v/v) was used as developing solvent. The silica gel $\mathrm{G}$ plates were finally visualized by sulfuric acid-phenol reagent (concentrated $\mathrm{H}_{2} \mathrm{SO}_{4}, 80 \%$ phenol solution) at $95{ }^{\circ} \mathrm{C}$ for $10 \mathrm{~min}$.

Fourier transform infrared spectroscopy (FTIR) analysis was also used to identify the functional groups of the rhamnolipids. $^{\text {20,22 }}$ A NICOLET380 FTIR spectrometer (Thermo Electron Corporation, USA) was used. The resolution was $0.5 \mathrm{~cm}^{-1}$. FTIR spectra were collected at wave numbers between $400 \mathrm{~cm}^{-1}$ and $4000 \mathrm{~cm}^{-1}$. Solid rhamnolipid product $(10 \mathrm{mg})$ was mixed with spectral purity $\mathrm{KBr}(100 \mathrm{mg})$. Then a translucent disc was made at $25 \mathrm{Mpa}$ for $30 \mathrm{~s}$.

\section{Structural composition analysis by HPLC-MS}

The three rhamnolipid products were respectively dissolved in $10 \%$ acetonitrile-water to a concentration of $500 \mathrm{mg} \mathrm{L}^{-1}$. Highpressure liquid chromatography-mass spectrometry (HPLC-MS) analysis was carried out according to previous studies. ${ }^{\mathbf{2 4 2 5}} \mathrm{A}$ liquid chromatography-mass spectrometer (Waters, Milford Massachusetts, USA) equipped with a reversed-phase C18 column $(\varnothing 2 \mathrm{~mm} \times 150 \mathrm{~mm} \times 0.5 \mu \mathrm{m})$ was used. The injection sample volume was $20 \mu \mathrm{L}$. The mobile phase was acetonitrilewater with gradient from $10 \%$ to $60 \%$. Mass spectrum scanning mass number ranged from $50 \mathrm{~m} / z$ to $800 \mathrm{~m} / \mathrm{z}$. Rhamnolipid congeners were identified based on $m / z^{24}$ Their relative proportions were calculated using the area normalization method.

\section{Bioactivity and stability analysis}

Three rhamnolipid products were respectively dissolved in distilled water to a concentration of $200 \mathrm{mg} \mathrm{L}^{-1}$. The rhamnolipid surface activity, emulsifying activity and stability against environmental stresses were evaluated. Surface tension was measured at $30{ }^{\circ} \mathrm{C}$ using a surface tension meter BZY-1 (Shanghai Hengping Instrument and Meter Factory, Shanghai, China). The emulsifying activity was measured as described previously. ${ }^{20}$ Crude oil sampled from the Xinjiang oil field was 
used. The emulsion index $\left(\mathrm{EI}_{24}\right)(\%)$ is defined as the height of the oil layer ( $\mathrm{mm}$ ) divided by the total height of the mixture (mm) and multiplied by $100 .^{20}$ Stability of rhamnolipids was evaluated under diverse environmental conditions. Rhamnolipid solutions were treated at different temperatures $\left(50{ }^{\circ} \mathrm{C}\right.$, $80^{\circ} \mathrm{C}, 100{ }^{\circ} \mathrm{C}$ and $\left.121^{\circ} \mathrm{C}\right)$, pH values $(2,4,6,8,10,11$ and 12$)$ and $\mathrm{NaCl}$ concentrations $(0 \%, 3 \%, 6 \%, 9 \%, 12 \%, 15 \%, 18 \%, 21 \%$ and $25 \%$ ) for $30 \mathrm{~min}$. The surface tension and $\mathrm{EI}_{24}$ were measured to evaluate the rhamnolipids' stability against environmental stresses.

The critical micelle concentration (CMC) of the rhamnolipid products was also measured. Rhamnolipid solutions with concentrations ranging from 0 to $120 \mathrm{mg} \mathrm{L}^{-1}$ were prepared. The surface tension of the solutions was measured. Curves of surface tension against rhamnolipid concentration were prepared. The concentration at the inflection point of the surface tension curve is the CMC of rhamnolipids.

\section{Oily sludge washing experiments}

The three rhamnolipid products were investigated for their capacity to remove oil from oily sludge. Experiments and analyses were performed as previously described. ${ }^{26}$ The oily sludge was sampled from an onshore oilfield in the northwest of China. It contained $13.66 \%$ total petroleum hydrocarbons (TPH). Using the extracted rhamnolipids and water, three kinds of rhamnolipid solutions $\left(200 \mathrm{mg} \mathrm{L}^{-1}\right)$ were prepared. In Erlenmeyer flasks, $10 \mathrm{~g}$ oily sludge was mixed with $100 \mathrm{~mL}$ rhamnolipid solution. Distilled water was used as negative control. The Erlenmeyer flasks were shaken at $180 \mathrm{rpm}$ and $60{ }^{\circ} \mathrm{C}$ for $24 \mathrm{~h}$ to wash the oily sludge. Then, samples were centrifuged at $5000 \mathrm{~g}$ for $10 \mathrm{~min}$ to separate the oil, water and oil sludge. The washed oil sludge samples were collected. Using tetrachloromethane, TPH in the oily sludge samples were extracted. The extraction liquid was collected and naturally dried at room temperature $\left(28{ }^{\circ} \mathrm{C}\right)$ in $90 \mathrm{~mm}$ plates. The weight of TPH was calculated, named as A. The weight of washed-out oil was the initial TPH amount in $10 \mathrm{~g}$ oily sludge $(1.366 \mathrm{~g}$ ) minus A. The washing efficiency (\%) was defined as the weight of washed-out oil divided by the initial TPH amount (1.366 g) multiplied by 100 .

\section{Results and discussion}

\section{Rhamnolipid production by the three strains using crude glycerol}

As shown in Fig. 1, all three strains can efficiently produce rhamnolipids using crude glycerol, decreasing the surface tension of the culture from $64 \mathrm{mN} \mathrm{m}^{-1}$ to $26 \mathrm{mN} \mathrm{m}^{-1}$. Using crude glycerol, strain SG produced $1.98 \mathrm{~g} \mathrm{~L}^{-1}$ of rhamnolipids (Fig. 1A), strain PrhlAB produced $2.87 \mathrm{~g} \mathrm{~L}^{-1}$ of rhamnolipids (Fig. 1B), and strain Rhl produced $0.87 \mathrm{~g} \mathrm{~L}^{-1}$ of rhamnolipids (Fig. 1C). The results demonstrated that crude glycerol can be used as a good carbon source by different rhamnolipidproducing strains.

The rhamnolipid concentrations in bacterial culture were determined by the oil-spreading method. ${ }^{23}$ The linear
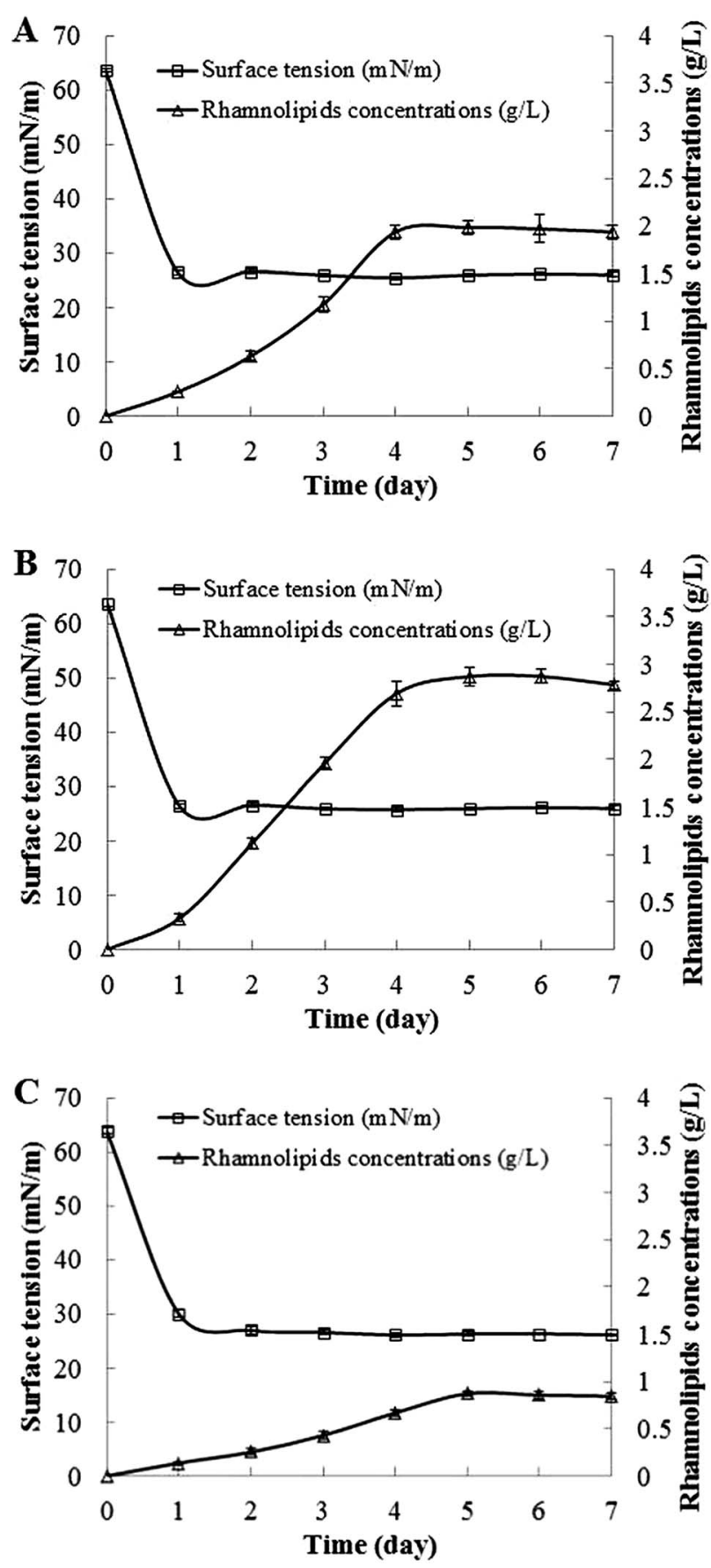

Fig. 1 Rhamnolipid production by strains SG (A), PrhlAB (B) and Rhl (C) grown on crude glycerol.

correlations for quantification of rhamnolipids produced by strain SG, PrhlAB and Rhl were as follows: $y=0.0624 x+5.4286$, $R^{2}=0.9908 ; y=0.0609 x+5.9643, R^{2}=0.9879 ; y=0.0602 x+$ $6.7857, R^{2}=0.9906$. In the linear correlations, $y(\mathrm{~mm})$ is the diameter of the oil-spreading circle, and $x\left(\mathrm{mg} \mathrm{L}^{-1}\right)$ is rhamnolipid concentrations in the bacterial culture. Values of $x$ are between $100 \mathrm{mg} \mathrm{L}^{-1}$ and $800 \mathrm{mg} \mathrm{L}^{-1}$. The linear correlations for quantification of three rhamnolipids were different, which indicated different bioactivity of the three rhamnolipid 
compositions, such as different emulsifying activity. ${ }^{23}$ So the structural compositions of the three rhamnolipids might be different. Rhamnolipids are a series of congeners composed of rhamnose and fatty acid. ${ }^{\mathbf{1 4 , 1 6}}$ Previous studies reported that different strains produced rhamnolipids with different compositions. ${ }^{\mathbf{1 4 2 4 , 2 7}}$ The industrial waste (crude glycerol) would be a low-cost substrate for producing different rhamnolipids products.

\section{TLC and FTIR analysis of the three rhamnolipid products}

All three biosurfactant samples formed yellow spots on TLC silica plates when stained with sulfuric acid-phenol reagent. TLC results showed that all three rhamnolipids contained reducing carbohydrates. The FTIR spectra of the three rhamnolipid products are shown in Fig. 2. The absorption bands around $2928 \mathrm{~cm}^{-1}, 2857 \mathrm{~cm}^{-1}$ and $1457 \mathrm{~cm}^{-1}$ were caused by the stretching vibrations of $\mathrm{C}-\mathrm{H}$ in aliphatic groups. The absorption band at $1731 \mathrm{~cm}^{-1}$ was caused by ester groups. These absorption bands were characteristic of rhamnolipids. These FTIR spectra are similar to the spectra of previously reported rhamnolipids. ${ }^{20,28}$ The TLC and FTIR analysis results confirmed that the three strains produced rhamnolipids using crude glycerol.

\section{Structural compositions of the three rhamnolipids products}

The liquid chromatogram results of the three rhamnolipids products are shown in Fig. 3. Because crude extracts of rhamnolipids products were used, some impurity peaks occurred in the chromatograms. According to Déziel's analysis methods, ${ }^{24}$ rhamnolipids produced by strain SG (Fig. 3A), PrhlAB (Fig. 3B) and Rhl (Fig. 3C) contained 8, 5 and 5 rhamnolipid congeners, respectively. As shown in Table 1, the rhamnolipid product of strain SG contained three mono-rhamnolipid and five dirhamnolipid congeners; the rhamnolipid product of strain PrhlAB contained four mono-rhamnolipid and one dirhamnolipid congeners; the five rhamnolipid congeners produced by strain Rhl were all mono-rhamnolipids. HPLC results confirmed that the three bacterial strains produced rhamnolipids using crude glycerol. Moreover, the structural

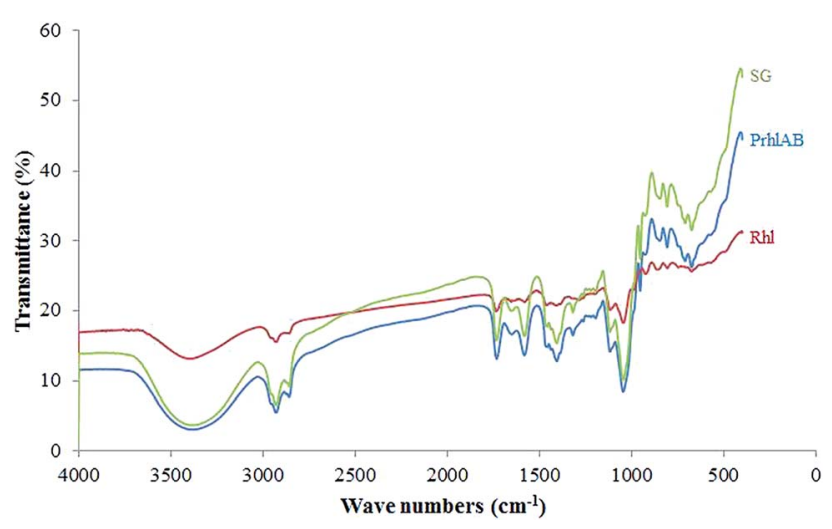

Fig. 2 Fourier transform infrared (FTIR) spectroscopy analysis of the three rhamnolipid products from strains SG, PrhlAB and Rhl. compositions of three rhamnolipid products were quite different. The proportions of mono-rhamnolipids in the rhamnolipid products of strains SG, PrhlAB and Rhl were $55.92 \%, 94.92 \%$ and $100 \%$, respectively.

\section{Emulsifying activity of the three rhamnolipid products}

As shown in Fig. 4, all three rhamnolipid solutions $\left(200 \mathrm{mg} \mathrm{L}^{-1}\right)$ emulsified crude oil with $\mathrm{EI}_{24}>60 \%$. Rhamnolipids produced by recombinant strain $\mathrm{Rhl}$ exhibited best emulsifying activity for crude oil, with $\mathrm{EI}_{24}=83.3 \%$. Only rhlAB genes were introduced into a non-rhamnolipid-producing strain DQ1 to construct recombinant strain $\mathrm{Rhl}^{22}$ Rhamnolipids produced by recombinant strain Rhl were all mono-rhamnolipids. Monorhamnolipids contain only one rhamnose. Di-rhamnolipids contain two rhamnoses. Mono-rhamnolipids are less hydrophilic relatively lipophilic than di-rhamnolipids. ${ }^{25}$ So monorhamnolipids have better emulsifying activity, whereas dirhamnolipids have better surface activity. ${ }^{25}$ In this study, the emulsifying activity for crude oil is positively related to the proportion of mono-rhamnolipids in the rhamnolipid products. Good emulsifying activity of biosurfactant is promising for bioremediation of hydrophobic pollutants. ${ }^{29,30}$

\section{Surface activity of the three rhamnolipid products}

As shown in Fig. 5, the surface tension first decreased with the increase of rhamnolipid concentrations. Then the surface tension remained constant with the increase of rhamnolipid concentrations. Rhamnolipids produced by wild-type strain SG decreased the water surface tension to $27.2 \mathrm{mN} \mathrm{m}^{-1}$. The critical micelle concentration (CMC) was $60 \mathrm{mg} \mathrm{L}^{-1}$ (Fig. 5A). Rhamnolipids produced by recombinant strain PrhlAB and strain Rhl also decreased the water surface tension to lower than $30.0 \mathrm{mN} \mathrm{m}^{-1}$. The CMCs of rhamnolipid mixtures produced by strain PrhlAB and strain Rhl were $80 \mathrm{mg} \mathrm{L}^{-1}$ (Fig. 5B) and $90 \mathrm{mg} \mathrm{L}^{-1}$ (Fig. 5C). Other studies have reported that the CMCs of rhamnolipids are in the range 40$150 \mathrm{mg} \mathrm{L}{ }^{-1} \cdot{ }^{14,31}$ Sodium dodecyl sulfate (SDS) is a commonly used synthetic surfactant. The CMC of SDS is $2100 \mathrm{mg} \mathrm{L}^{-1} \cdot{ }^{32,33}$ Compared with synthetic surfactants, the three rhamnolipid products are highly excellent.

The hydrophilic moiety of di-rhamnolipids contains two rhamnoses. Di-rhamnolipids exhibit are more hydrophilic than mono-rhamnolipids. Previous studies also reported that dirhamnolipids had stronger surface activity than mono-rhamnolipids. ${ }^{25,34}$ Rhamnolipids produced by strain SG contained the most abundant di-rhamnolipids. So the rhamnolipids produced by strain SG exhibited the best surface activity, such as lowest CMC and surface tension. Oher studies have also reported that mono-rhamnolipids, containing only one rhamnose (hydrophilic moiety), are less soluble and adsorb to surfaces more strongly, so they have higher CMC for hydrocarbon solubilization than di-rhamnolipids. ${ }^{35}$ The rhamnolipids produced by strain Rhl were all mono-rhamnolipids. So this product showed highest CMC and surface tension value. 

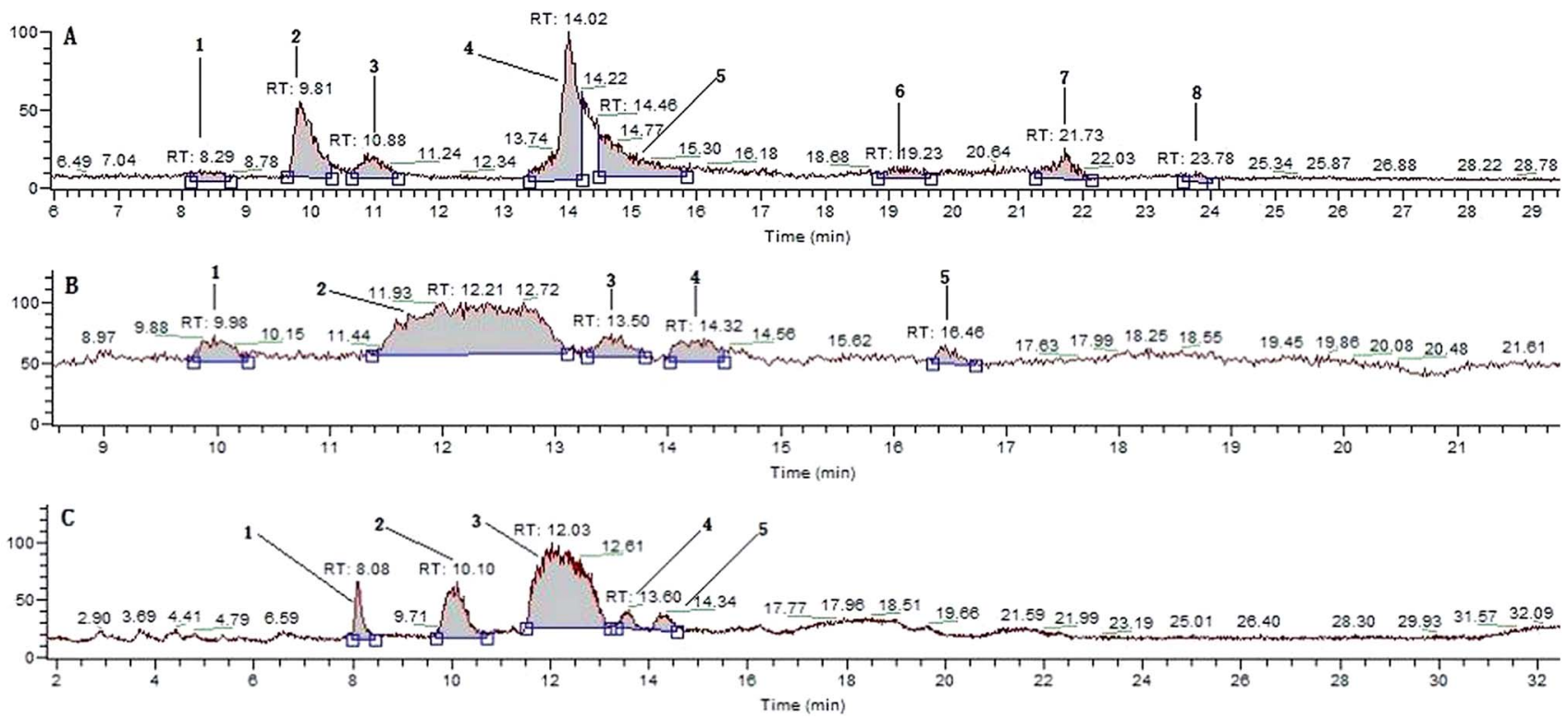

Fig. 3 Liquid chromatogram results for the three rhamnolipid products: (A) rhamnolipids produced by strain SG, (B) rhamnolipids produced by strain PrhIAB, (C) rhamnolipids produced by strain Rhl.

\section{Stability of the three rhamnolipid products}

The three rhamnolipids retained their same properties after treating at $121{ }^{\circ} \mathrm{C}$ for $30 \mathrm{~min}$, which indicated that the three rhamnolipids were thermostable. In the presence of $15 \%$ of $\mathrm{NaCl}$, all three rhamnolipids solutions had surface tension lower than $30.0 \mathrm{mN} \mathrm{m}^{-1}$ and emulsified crude oil with $\mathrm{EI}_{24}$ higher than $60.0 \%$. These results revealed that the three rhamnolipids were salt-tolerant. After treatment with different $\mathrm{pH}$ values in the range 4-10, all three rhamnolipids could decrease the surface tension to lower than $35.0 \mathrm{mN} \mathrm{m}^{-1}$ and emulsify crude oil with $\mathrm{EI}_{24}$ higher than $60.0 \%$. The three rhamnolipids are stable at temperatures lower than $121^{\circ} \mathrm{C}, \mathrm{pH}$ values 4-10 and salinities lower than 15\% NaCl. Therefore, all three rhamnolipids can potentially be used in complex and extreme environments, such as oil reservoirs and petroleumcontaminated soil.

\section{Enhanced oil removal from oily sludge by the three rhamnolipid products}

The initial total petroleum hydrocarbons (TPH) amount in oily sludge is $13.66 \%$. After washing with rhamnolipids of strains SG, PrhlAB and Rhl, the TPH amounts in the washed oily sludge samples were $9.47 \%, 7.11 \%$ and $6.31 \%$, respectively. High concentrations of petroleum hydrocarbons in soil are toxic to

Table 1 Structural composition of rhamnolipids produced by strains SG, PrhlAB and Rhl

\begin{tabular}{|c|c|c|c|c|c|}
\hline Strain & $\begin{array}{l}\text { Chromatographic } \\
\text { peak number }\end{array}$ & $\begin{array}{l}\text { Retention time } \\
\text { (min) }\end{array}$ & Mass spectrum signal $(\mathrm{m} / \mathrm{z})$ & $\begin{array}{l}\text { Rhamnolipid } \\
\text { homologue }\end{array}$ & $\begin{array}{l}\text { Relative abundance } \\
(\%)\end{array}$ \\
\hline \multirow[t]{8}{*}{ SG } & 1 & 8.29 & 447 & Rha- $\mathrm{C}_{8}-\mathrm{C}_{8}$ & 4.24 \\
\hline & 2 & 9.81 & 475 & Rha- $\mathrm{C}_{8}-\mathrm{C}_{10}$ & 19.47 \\
\hline & 3 & 10.88 & 621 & Rha-Rha- $\mathrm{C}_{8}-\mathrm{C}_{10}$ & 7.77 \\
\hline & 4 & 14.02 & 503 & Rha- $\mathrm{C}_{10}-\mathrm{C}_{10}$ & 32.22 \\
\hline & 5 & 14.46 & 649 & Rha-Rha- $\mathrm{C}_{10}-\mathrm{C}_{10}$ & 21.25 \\
\hline & 6 & 19.23 & 677 & Rha-Rha- $\mathrm{C}_{10}-\mathrm{C}_{12}$ & 4.71 \\
\hline & 7 & 21.73 & 675 & Rha-Rha- $\mathrm{C}_{10}-\mathrm{C}_{12: 1}$ & 7.85 \\
\hline & 8 & 23.78 & 705 & Rha-Rha- $\mathrm{C}_{12}-\mathrm{C}_{12}$ & 2.50 \\
\hline \multirow[t]{5}{*}{ PrhlAB } & 1 & 9.98 & 475 & Rha- $\mathrm{C}_{8}-\mathrm{C}_{10}$ & 9.50 \\
\hline & 2 & 12.21 & 503 & Rha- $\mathrm{C}_{10}-\mathrm{C}_{10}$ & 64.58 \\
\hline & 3 & 13.50 & 529 & Rha- $\mathrm{C}_{10}-\mathrm{C}_{12: 1}$ & 11.02 \\
\hline & 4 & 14.32 & 531 & Rha- $\mathrm{C}_{10}-\mathrm{C}_{12}$ & 9.83 \\
\hline & 5 & 16.46 & 649 & Rha-Rha- $\mathrm{C}_{10}-\mathrm{C}_{10}$ & 5.08 \\
\hline \multirow[t]{5}{*}{ Rhl } & 1 & 8.08 & 447 & Rha- $\mathrm{C}_{8}-\mathrm{C}_{8}$ & 6.46 \\
\hline & 2 & 10.10 & 475 & Rha- $\mathrm{C}_{8}-\mathrm{C}_{10}$ & 15.84 \\
\hline & 3 & 12.03 & 503 & Rha- $\mathrm{C}_{10}-\mathrm{C}_{10}$ & 68.55 \\
\hline & 4 & 13.60 & 529 & Rha- $\mathrm{C}_{10}-\mathrm{C}_{12: 1}$ & 4.56 \\
\hline & 5 & 14.34 & 531 & Rha- $\mathrm{C}_{10}-\mathrm{C}_{12}$ & 4.58 \\
\hline
\end{tabular}




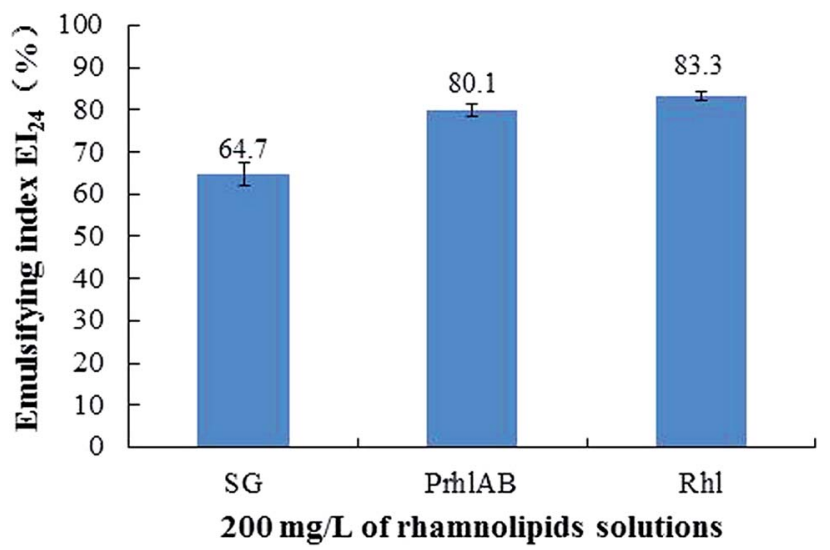

Fig. 4 Emulsifying activity of the three rhamnolipid products from strains SG, PrhlAB and Rhl.
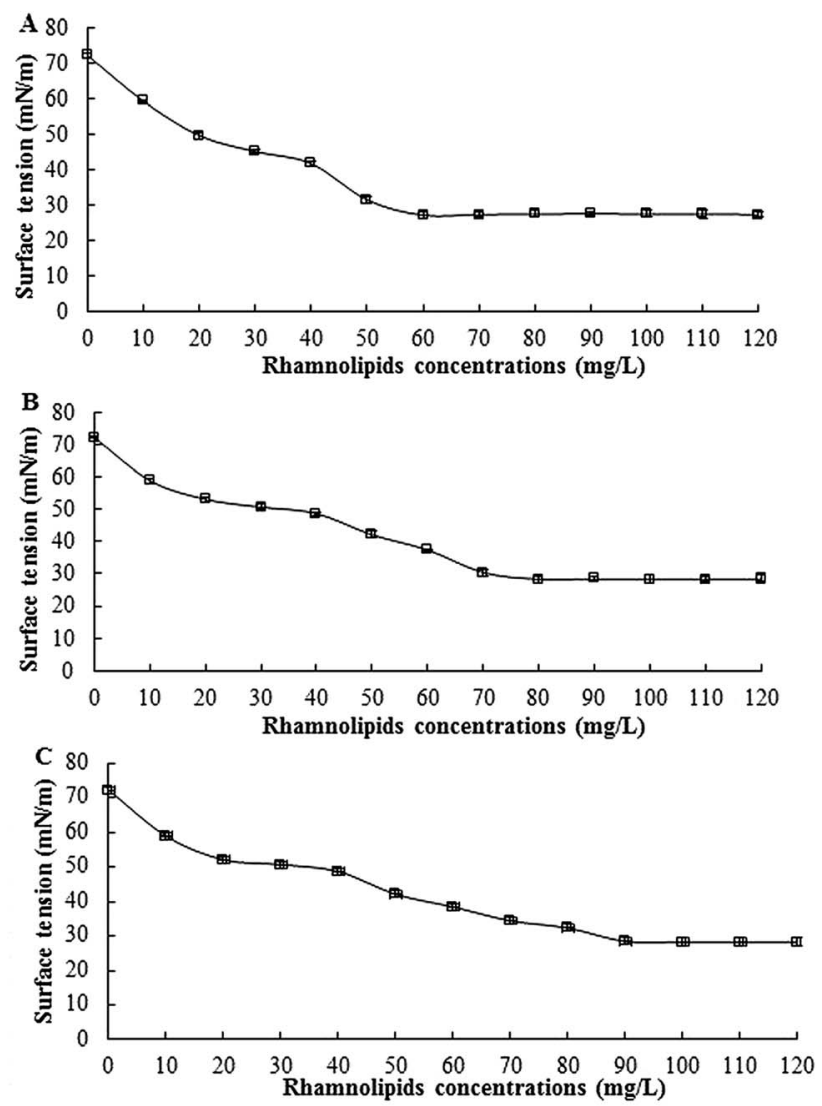

Fig. 5 Critical micelle concentration determination based on relationship graphs between surface tension and rhamnolipid concentrations: (A) rhamnolipids produced by strain SG, (B) rhamnolipids produced by strain PrhlAB, (C) rhamnolipids produced by strain Rhl.

microbes. ${ }^{36,37}$ The rhamnolipid washing process significantly reduced the TPH amount in oily sludge, which was beneficial to microbial remediation of oily sludge. The washing efficiency of the three rhamnolipid products from strains SG, PrhlAB and Rhl were $30.67 \%, 47.95 \%$ and $53.81 \%$, respectively. The washing efficiency of distilled water was $8.20 \%$. Compared with distilled water, all three rhamnolipid solutions $\left(200 \mathrm{mg} \mathrm{L}^{-1}\right)$ efficiently removed TPH from oily sludge. Previous studies reported that biosurfactants are excellent agents for oily sludge washing: ${ }^{38-40}$ Another study also showed that biosurfactants (200 $\mathrm{mg} \mathrm{L}^{-1}$ ) produced by a Bacillus subtilis strain removed $62 \%$ of TPH from contaminated soil. ${ }^{26}$ In the present study, monorhamnolipids produced by recombinant strain Rhl exhibited the best washing efficiency for oily sludge. Mono-rhamnolipids had better emulsifying activity. The results indicated that mono-rhamnolipids are excellent agents for oily sludge washing.

\section{Perspectives}

A previous study showed that glycerol, which has good solubility in water, can be easily absorbed and metabolized by microorganisms. ${ }^{22}$ Moreover, crude glycerol is the main by-product of biodiesel and saponification processess. ${ }^{41,42}$ In this study, crude glycerol was used as a cheap carbon source for rhamnolipid production by different strains. Therefore, crude glycerol is a promising and inexpensive carbon source for rhamnolipid production. Rhamnolipids are a mixture, consisting of one or two rhamnoses (hydrophilic moiety) and one or two $\beta$-hydroxy fatty acids with different lengths (hydrophobic moiety). ${ }^{16}$ Different bacterial strains, culture media and cultivation conditions produce rhamnolipid mixtures with different types and proportions of congeners. ${ }^{18,43}$ Their physico-chemical properties and applications depend on the structural compositions of the rhamnolipid mixtures. ${ }^{14}$ In this study, the emulsifying activity for crude oil is positively related to the proportion of mono-rhamnolipids in the rhamnolipid products. Good emulsifying activity of biosurfactant is promising for bioremediation of hydrophobic pollutants. ${ }^{29,30}$ In this study, mono-rhamnolipids exhibited the best washing efficiency for oily sludge. Therefore, future research should concentrate on ways to enhance mono-rhamnolipid production, such as biosynthesis pathway regulation and medium optimization.

\section{Conclusions}

Industrial waste (crude glycerol) can be used for rhamnolipid production using three different bacterial strains: Pseudomonas aeruginosa SG, PrhlAB and Rhl. The three strains produce different rhamnolipid mixtures. The proportions of monorhamnolipids in the rhamnolipid products from strains SG, PrhlAB and Rhl are 55.92\%, 94.92\% and 100\%, respectively. The three rhamnolipid products are thermostable and salt-tolerant. The emulsifying activity for crude oil is positively related to the proportion of mono-rhamnolipids in the rhamnolipid products. The surface activity is positively related to the proportion of dirhamnolipids. All three rhamnolipid products efficiently removed TPH from oily sludge. Mono-rhamnolipids exhibited the best washing efficiency for oily sludge.

\section{Conflicts of interest}

There are no conflicts to declare. 


\section{Acknowledgements}

This work was financially supported by the National Natural Science Foundation of China (31700117), the China Postdoctoral Science Foundation (2017M621292) and the Open Fund of the Key Laboratory of Pollution Ecology and Environmental Engineering, Chinese Academy of Sciences.

\section{References}

1 G. Hu, J. Li and G. Zeng, J. Hazard. Mater., 2013, 261, 470490.

2 C. Liu, Y. Zhang, S. Sun, L. Huang, L. Yu, X. Liu, R. Lai, Y. Luo, Z. Zhang and Z. Zhang, J. Pet. Sci. Eng., 2018, 170, 14-20.

3 R. D. C. F. Silva, D. G. Almeida, R. D. Rufino, J. M. Luna, V. A. Santos and L. A. Sarubbo, Int. J. Mol. Sci., 2014, 15(7), 12523-12542.

4 Y. X. Gao, R. Ding, X. Chen, Z. B. Gong, Y. Zhang and M. Yang, Ultrasonics, 2018, 90, 1-4.

5 B. Lin, J. Wang, Q. Huang and Y. Chi, Fuel, 2017, 200, 124133.

6 X. Mao, R. Jiang, W. Xiao and J. Yu, J. Hazard. Mater., 2015, 285, 419-435.

7 Y. Zhang, Q. Zhao, J. Jiang, K. Wang, L. Wei, J. Ding and H. Yu, Bioresour. Technol., 2017, 243, 820-827.

8 I. M. Banat, R. S. Makkar and S. S. Cameotra, Appl. Microbiol. Biotechnol., 2000, 53, 495-508.

9 J. S. Clifford, M. A. Ioannidis and R. L. Legge, J. Colloid Interface Sci., 2007, 305(2), 361-365.

10 G. Liu, H. Zhong, X. Yang, Y. Liu, B. Shao and Z. Liu, Biotechnol. Bioeng., 2018, 115(4), 796-814.

11 B. Doshi, M. Sillanpää and S. Kalliola, Water Res., 2018, 135, 262-277.

12 M. P. Płociniczak, G. A. Płaza, G. Piotrowska-Seget and S. S. Cameotra, Int. J. Mol. Sci., 2011, 12, 633-654.

13 R. M. Maier, Adv. Appl. Microbiol., 2003, 52, 101-121.

14 A. M. Abdel-Mawgoud, F. Lépine and E. Déziel, Appl. Microbiol. Biotechnol., 2010, 86, 1323-1336.

15 N. Roongsawang, K. Washio and M. Morikawa, Int. J. Mol. Sci., 2010, 12(1), 141-172.

16 M. M. Müller, J. H. Kügler, M. Henkel, M. Gerlitzki, B. Hörmann, M. Pöhnlein, C. Syldatk and R. Hausmann, J. Biotechnol., 2012, 162, 366-380.

17 T. Tiso, R. Zauter, H. Tulke, B. Leuchtle, W. J. Li, B. Behrens, A. Wittgens, F. Rosenau, H. Hayen and L. M. Blank, Microb. Cell Fact., 2017, 16(1), 225.

18 L. Zhang, J. E. Pemberton and R. M. Maier, Process Biochem., 2014, 49, 989-995.

19 M. Benincasa, A. Abalos, I. Oliveira and A. Manresa, Antonie van Leeuwenhoek, 2004, 85(1), 1-8.

20 F. Zhao, J. Zhang, R. Shi, S. Han, F. Ma and Y. Zhang, RSC Adv., 2015, 5, 36044-36050.
21 F. Zhao, Q. Cui, S. Han, H. Dong, J. Zhang, F. Ma and Y. Zhang, RSC Adv., 2015, 5(86), 70546-70552.

22 F. Zhao, R. Shi, J. Zhao, G. Li, X. Bai, S. Han and Y. Zhang, J. Appl. Microbiol., 2015, 118, 379-389.

23 F. Zhao, X. Liang, Y. Ban, S. Han, J. Zhang, Y. Zhang and F. Ma, Tenside, Surfactants, Deterg., 2016, 53(3), 243-248.

24 E. Déziel, F. Lépine, D. Dennie, D. Boismenu, O. A. Mamer and R. Villemur, Biochim. Biophys. Acta, Mol. Cell Biol. Lipids, 1999, 1440(2), 244-252.

25 F. Zhao, R. Shi, F. Ma, S. Han and Y. Zhang, Microb. Cell Fact., 2018, 17(1), 39.

26 C. C. Lai, Y. C. Huang, Y. H. Wei and J. S. Chang, J. Hazard. Mater., 2009, 167(1-3), 609-614.

27 E. Haba, A. Abalos, O. Jauregui, M. J. Espuny and A. Manresa, J. Surfactants Deterg., 2003, 6(2), 155-161.

28 A. J. Das and R. Kumar, Bioresour. Technol., 2018, 260, 233240.

29 E. J. Silva, P. F. Correa, D. G. Almeida, J. M. Luna, R. D. Rufino and L. A. Sarubbo, Colloids Surf., B, 2018, 172, 127-135.

30 Z. Zeng, Y. Liu, H. Zhong, R. Xiao, G. Zeng, Z. Liu, M. Cheng, C. Lai, C. Zhang, G. Liu and L. Qin, Sci. Total Environ., 2018, 634, 1-11.

31 E. J. Gudiña, A. I. Rodrigues, E. Alves, M. R. Domingues, J. A. Teixeira and L. R. Rodrigues, Bioresour. Technol., 2015, 177, 87-93.

32 H. Yin, J. Qiang, Y. Jia, J. S. Ye, H. Peng, H. M. Qin, N. Zhang and B. Y. He, Process Biochem., 2009, 44, 302-308.

33 J. G. D. Oliveira and C. H. Garcia-Cruz, Braz. Arch. Biol. Technol., 2013, 56(1), 155-160.

34 X. Zhang, Q. Guo, Y. Hu and H. Lin, Chemosphere, 2013, 90(2), 581-587.

35 A. Perfumo, I. M. Banat, F. Canganella and R. Marchant, Appl. Microbiol. Biotechnol., 2006, 72(1), 132-138.

36 D. Sarkar, M. Ferguson, R. Datta and S. Birnbaum, Environ. Pollut., 2005, 136(1), 187-195.

37 V. Labud, C. Garcia and T. Hernandez, Chemosphere, 2007, 66(10), 1863-1871.

38 O. A. Johnson and A. C. Affam, Environ. Eng. Res, 2019, 24(2), 191-201.

39 P. Yan, M. Lu, Q. Yang, H. L. Zhang, Z. Z. Zhang and R. Chen, Bioresour. Technol., 2012, 116, 24-28.

40 A. Roy, A. Dutta, S. Pal, A. Gupta, J. Sarkar, A. Chatterjee and S. K. Kazy, Bioresour. Technol., 2018, 253, 22-32.

41 G. P. da Silva, M. Mack and J. Contiero, Biotechnol. Adv., 2009, 27, 30-39.

42 S. N. R. L. Silva, C. B. B. Farias, R. D. Rufino, J. M. Luna and L. A. Sarubbo, Colloids Surf., B, 2010, 79, 174-183.

43 Z. A. Raza, Z. M. Khalid and I. M. Banat, J. Environ. Sci. Health, Part A: Toxic/Hazard. Subst. Environ. Eng., 2009, 44, 1367-1373. 